\title{
A robust-coded pattern projection for dynamic 3D scene measurement
}

\author{
J. Salvi ${ }^{\text {a,* }}$, J. Batlle ${ }^{\text {a,1 }}$, E. Mouaddib ${ }^{\text {b,2 }}$ \\ ${ }^{a}$ Computer Vision and Robotics Group, Institute of Informatics and Applications, University of Girona, Avda. Lluis Santaló s/n, 17071 \\ Girona, Spain \\ b Automatic Systems Laboratory, University of Picardie-Jules Verne, 7, Rue du Moulin Neuf, 80000 Amiens, France \\ Received 11 September 1997; received in revised form 29 May 1998
}

\begin{abstract}
This paper presents a new coded structured light pattern which permits to solve the correspondence problem by a single shot and without using geometrical constraints. The pattern is composed by the projection of a grid made by coloured slits in such a way that each slit with its two neighbours appears only once in the pattern. The technique proposed permits a rapid and robust 3D scene measurement, even with moving objects. (ㄷ 1998 Elsevier Science B.V. All rights reserved.
\end{abstract}

Keywords: Coded pattern projection; Correspondence problem; Structured light; 3D scene measurement

\section{Introduction}

There are some essential problems in stereo vision which make it difficult to solve the correspondence problem between both image planes. Some authors consider to solve the problem of matching from singular points, i.e. finding points in both image planes with more or less the same neighbourhood characteristics, classifying them as corners and vertexes. Since both 2D images are projections of the same scene, images taken from different positions may be quite different one from the other. Consequently, this hypothesis is rather poor and becomes even poorer if we take into

\footnotetext{
${ }^{*}$ Corresponding author. Tel.: +34 7241 8474; fax: +34 7241 8098; e-mail: qsalvi@eia.udg.es.

${ }^{1}$ E-mail: jbatlle@eia.udg.es.

2 E-mail: Mustapha.Mouaddib@u-picardie.fr.
}

account that the precision of the $3 \mathrm{D}$ measurement depends on the distance between both cameras. The more this distance increases, the more the measurement will be precise. However, when one increases the distance between both cameras, the projective images of the $3 \mathrm{D}$ scenes become more different, constraining considerably their match using similarity. Of course, we must also consider that in the same 3D scene more than one object with approximately the same shape and size could be present. In this case it becomes rather difficult to match the images from the mere concept of singular points. However, some geometrical constraints can be used to reduce the problem of matching. One of the most popular constraints is known as the epipolar constraint imposed by the geometrical relationship between both cameras (Faugeras, 1993, p. 169). The epipolar constraint allows us to reduce the searching of the 
correspondence from the two-dimensional space of the image plane into the one-dimensional one of the epipolar line. However, we must be aware that some points in an image plane might not have a correspondence on the other one due to a surface occlusion or simply because it has been projected out of the scope of the camera. Note that we are unable to know a priori whether a point has a correspondence or not, which makes the matching enormously difficult and imposes the use of a posterior step to remove false matching mostly by using a region growing algorithm. At this point another constraint which arises from the structure of the objects of the scene can be used. It could be assumed that the scene surfaces vary in depth smoothly almost everywhere. This constraint is known as the disparity gradient. Although this constraint can be used to reduce false matches obtained from the geometrical properties of the epipolar line, the reader must know that it cannot be used at depth discontinuities. Note that depth discontinuities principally produce the edges and vertexes that will be used as tokens to the matching process. We must also note that almost all stereo vision systems restrict the obtaining of 3D information from the vertexes and corners of the objects. Hence the disparity gradient could not be used except if we obtain 3D information following the edges which definitely complicates the matching process increasing the computing time.

It is known that the correspondence problem can be alleviated leaving off stereo vision, and going to the structured light concept. Here, the second stereo camera is replaced by a light source, which projects a known pattern of light on the measuring scene. The first stereo camera images the illuminated scene and, analysing the deformations of the imaged pattern with respect to the projected one, can obtain the desired 3D information. Of course, depending on the chosen pattern, some correspondences between the projected pattern and the imaged one should be solved. Most of the proposed structured light techniques are based on the projection of regular patterns on the measuring scene (e.g. Will and Pennington, 1971; Hu and Stockman, 1989; Wang and Pandey, 1991). All these methods obtain 3D information from the geometric constraint propagation, espe- cially from the epipolar constraint, and some of them are rather limited to measure surfaces with depth discontinuities.

In recent years a new structured light technique has increased in importance. This technique is based on a unique codification of each token of light projected on the scene. When the token is imaged by the camera, this codification allows us to obtain the correspondence, i.e. to know where it comes from. Then 3D measurements are directly obtained as we are not to use hard computational geometric constraints. This technique is basically known as coded structured light. Several coded structured light techniques have been proposed in the past, which have been discussed and compared in a quite recent survey (Batlle et al., 1997). The techniques are mostly based on coded dot projection (e.g. Vuylsteke and Oosterlinck, 1990; Yee and Griffin, 1994; Ito and Ishii, 1995) and coded slit projection (e.g. Boyer and Kak, 1987; Tajima and Iwakawa, 1990; Maruyama and Abe, 1993).

Our goal is to propose a new coded structured light pattern. The technique is based on obtaining 3D scene information from a single pattern shot projection, so that it could be used to measure static and dynamic scenes. The projecting pattern has been designed such that it could be easily and robustly segmented, allowing us to efficiently solve the correspondence problem without spending a lot of computing time.

The paper is divided as follows. Firstly, the new pattern is presented. Secondly, the system modelling and the iterative calibration method are explained. Then, the segmentation process and some experimental results are shown and discussed. Finally, conclusions are presented.

\section{Pattern design}

Our intention is to perform the measurement of any static or dynamic scene by the projection of a coded structured light pattern on the measuring objects. Therefore, a single pattern shot projection is allowed as the scene could be composed by moving objects. Furthermore, the pattern must be segmented without a significant amount of computing time. If we project a straight line on planar 
surfaces, straight segments of such line will be imaged leading to an easy segmentation. That is why we propose to use a pattern based on the projection of straight lines. Several authors have proposed striped pattern projection coded along a single axe (e.g. Boyer and Kak, 1987; Tajima and Iwakawa, 1990), because a single axe codification of the projected pattern is needed to infer the 3D information from the $2 \mathrm{D}$ points grabbed by the camera (to demonstrate this deduction the reader is referred to (Hall et al., 1982)). Nevertheless, we suggest to improve the robustness of the matching using a grid pattern projection coded along both axes. 3D information is inferred from the leastsquares method. Quite a few techniques based on both axes codification have been proposed. For instance, Griffin et al. (1992) proposed to project uniquely encoded dots. The code of a given dot is made by the colour of that dot and of its four neighbours. However, due to an absence of links among dot neighbours, the identification of the neighbours of a given dot is highly determined by the measuring scene. Two years later, Yee and Griffin (1994) used the same principle of codification to project uniquely encoded binary dots. The dots were linked, making easy the identification of the neighbours. However, the segmentation of the pattern and further dot identification become harder as dots are coded by using different shapes. That is the reason why we propose to use a grid pattern which may be easily segmented.

We propose to code the grid by colouring its horizontal and vertical slits. Although it is known that high saturated colour objects may produce further segmentation errors, we assume that they are not abundant in the scene. We must also note that a system based on light projection is mostly to be used under scene light control. Whereas only the light coming from the pattern is allowed if a good segmentation is required, slender light is permitted if a high power projector lamp is used. Note that slender light will not produce the albedo effect which could make difficult the segmentation of the pattern.

We propose to project a grid made by coloured slits. Although the pattern has been printed in greyscale in Fig. 1, it is a coloured one. Six different colours have been used. As an example we

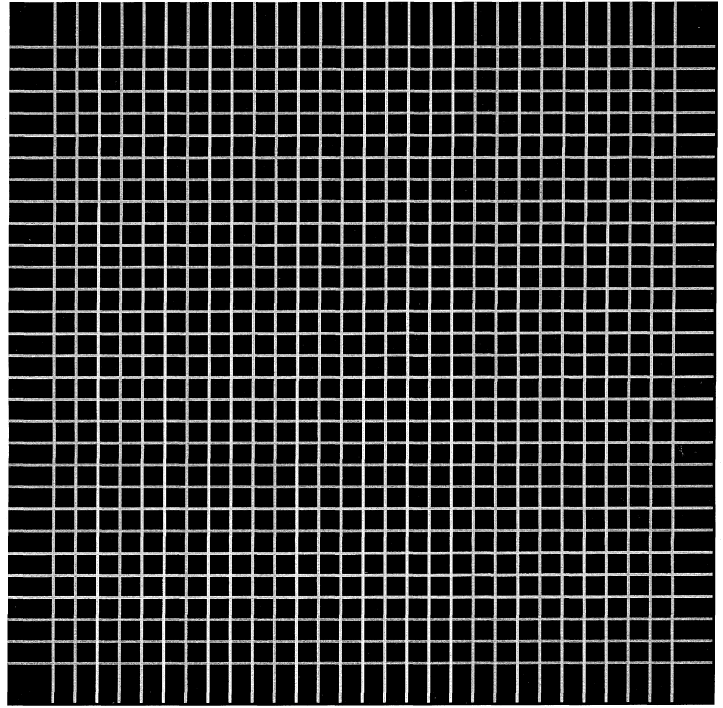

Fig. 1. The coloured pattern.

have chosen red, green and blue to code the horizontal slits; and magenta, cyan and yellow to code the vertical ones. But, what is important is that we must chose well-spaced colours in the HSI cone, through which they can be well segmented using their hue and saturation components. We have used a codification similar to the one used by Griffin et al. (1992). More precisely, Griffin et al. are interested in obtaining the uniquely coded maximum matrix from a determined basis. We are only interested in the maximum vector, that is, the maximum sequence of triplets that can be made without repetition. Given a basis $p$, the maximum vector is defined by a sequence of numbers in the $1 \ldots p$ range, as shown by Eq. (1).

The problem can be solved using the graph theory. Given a $p$ basis we can obtain a set of nodes $S$ where each node is defined by a triplet of three elements of $P=\{1,2, \ldots, p\}$, that is $S=V R_{p}{ }^{3}$ obtaining up to $p^{3}$ nodes. A node is defined by a triplet $i j k \mid i, j, k \in P$. At this point, the graph can be constructed. Each node $N=i j k$ has $p$ inputs and $p$ outputs, but the nodes where $i=j=k$ as they have $p-1$ inputs and $p-1$ outputs. Then, the problem is reduced to construct the sequence of triplets by visiting all the nodes of the graph only once, which can be easily solved by graph 
theory. Note that we can go from the node $N=i j k$ to the node $N^{\prime}=i^{\prime} j^{\prime} k^{\prime}$ only if $j=i^{\prime}$ and $k=j^{\prime}$. (For a more detailed explanation the reader is referred to Griffin et al., 1992, p. 611.)

In our example, we have chosen a basis equal to 3 , then we obtain a sequence of 29 numbers in the range $1 \ldots 3$. This sequence defines a coloured slit sequence. Horizontal slits are obtained substituting 1, 2 and 3 by red, green and blue, respectively; and the vertical slits substituting 1, 2 and 3 by magenta, cyan and yellow. What is important is that each slit colour with its two neighbour slits colours forms a triplet that exists only once in the whole pattern. Slits are constantly spaced forming a regular grid of $29 \times 29$ cross-points. The resolution of the pattern may be increased by simply changing the $p$ basis, i.e. using more colour primitives.

In the aim of testing the proposed pattern, a lab scenario has been set (see Fig. 2). The system is composed by an RGB camera, a computer and an electronic slide projector. In Fig. 2, the camera calibrating pattern is also shown. The coloured pattern is shaped in a $512 \times 512$ RGB image which is projected on the measuring scene using the electronic slide projector, and the scene is then captured by the camera into the computer memory.

\section{Calibration}

The hard calibration method is based on obtaining the intrinsic and extrinsic parameters of the camera model and the projecting system model knowing the coordinates of the $3 \mathrm{D}$ object points. In fact, the problem is reduced to the computation of the transformation equations which models the relation between the 3D object points $\left(x_{r}, y_{r}, z_{r}\right)$ and their 2D observable correspondence point $\left(u_{k}\right.$, $v_{k}$ ) in the image plane of the camera (or their 2D projecting points of the projector system). The linear relation is modelled by a $3 \times 4$ transformation matrix. This matrix contains the 6 extrinsic parameters defined by the three rotation angles $(\alpha$, $\beta, \gamma)$, expressed as a $3 \times 3$ rotation matrix $\boldsymbol{R}$, and the translation vector $t=\left(t_{x}, t_{y}, t_{z}\right)$; and the 4 intrinsic parameters defined by the projection of the optical centre in the image plane (or projector frame) $\left(u_{0}, v_{0}\right)$, and the perspective parameters $\left(\alpha_{u}, \alpha_{v}\right)$ (the reader is referred to (Toscani, 1987) for a wide explanation). However, as a result of some types of imperfections in the design and assembly of the lens composing the optical system, a linear relation does not hold true (see Tsai, 1987). These kinds of imperfections, known as lens distortion, can be modelled by a radial and tangential approximation. Radial distortion causes an

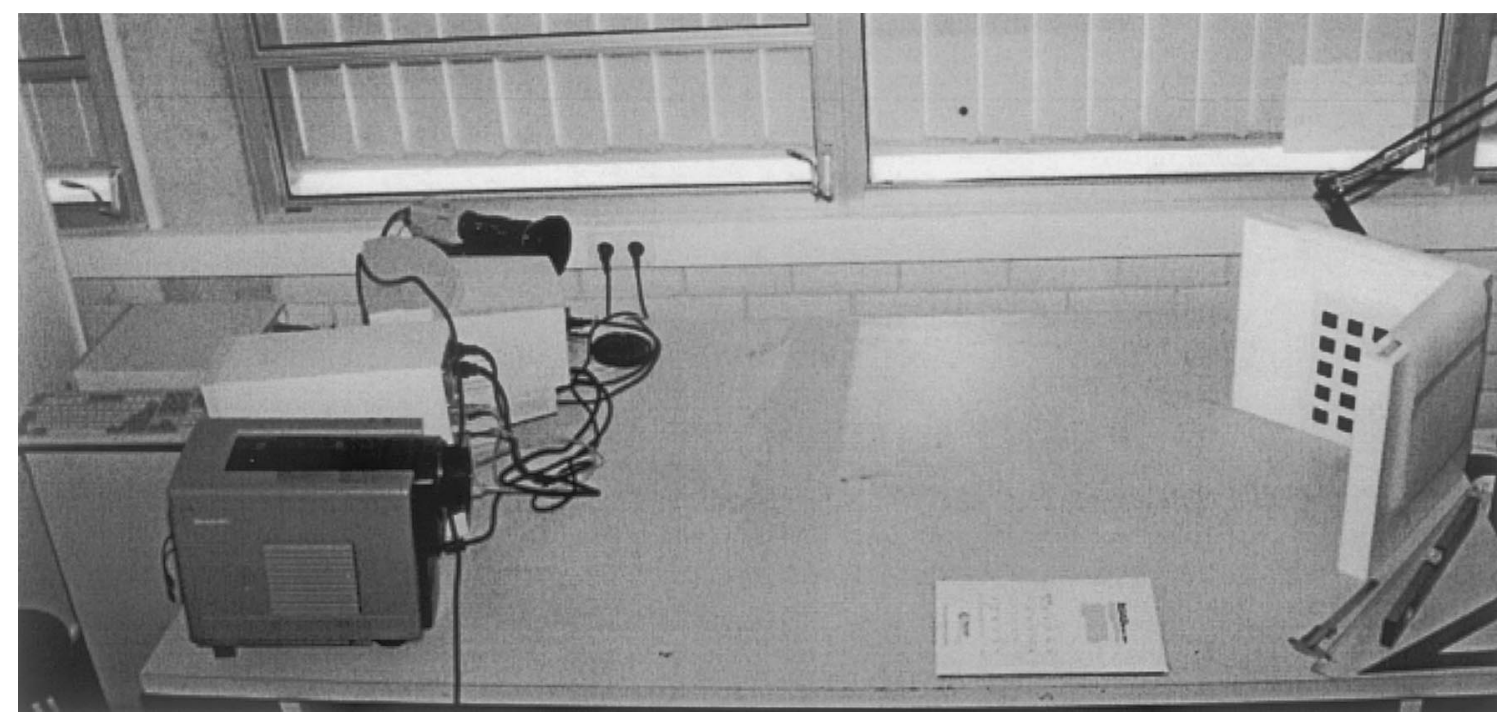

Fig. 2. The scenario of the 3D measuring system. 
inward or outward displacement of a given image point from its ideal location, and it has been demonstrated to be quite important in camera modelling (see (Weng and Cohen, 1992) for a detailed explanation). Radial lens distortion can be approximated by nonlinear equations governed by the first coefficient $k_{1}$. The relation between a 3D object point and its observable image point (or projective point) is then modelled by Eqs. (1) and (2).

$U=X_{u}-X_{d}-k_{1} r^{2} X_{d}$,

$V=Y_{u}-Y_{d}-k_{1} r^{2} Y_{d}$,

where

$$
\begin{aligned}
& \left(\begin{array}{c}
X \\
Y \\
Z
\end{array}\right)=\left[\begin{array}{ll}
\boldsymbol{R} & t
\end{array}\right]\left(\begin{array}{c}
x_{r} \\
y_{r} \\
z_{r} \\
1
\end{array}\right), \quad r^{2}=X_{d}^{2}+Y_{d}^{2}, \\
& X_{u}=f \frac{X}{Z}, \quad Y_{u}=f \frac{Y}{Z} \\
& X_{d}=\frac{\left(u_{k}-u_{0}\right)}{k_{u}}, \quad Y_{d}=\frac{\left(v_{k}-v_{0}\right)}{k_{v}},
\end{aligned}
$$

we have expanded the $\alpha_{u}$ and $\alpha_{v}$ parameters as follows:

$$
\alpha_{u}=f k_{u}, \quad \alpha_{v}=f k_{v} .
$$

Let us consider Eqs. (1) and (2) like two functions $\boldsymbol{G}$ which depend on 11 unknowns. These 11 unknowns are arranged in a vector called $\boldsymbol{X}$, that is $\boldsymbol{X}=\left\{\boldsymbol{u}_{0}, \boldsymbol{u}_{1}, \ldots, \boldsymbol{u}_{10}\right\}$. The obtaining of the $\boldsymbol{X}$ vector is the main objective of the calibrating method. As a result of the inclusion of the lens distortion in the camera model, $\boldsymbol{G}$ has become a nonlinear equation. That is why we have to use an iterative calibrating method to minimise $\boldsymbol{G}$ in order to obtain $\boldsymbol{X}$.

We want to calculate the values of $\boldsymbol{X}$ which solve the equation $\boldsymbol{G}(\boldsymbol{X})=0$ iterating from an initial solution $\boldsymbol{X}_{0}$. The initial solution is computed using the method of Toscani assuming no lens distortion $\left(k_{1}=0\right)$. We have used the widely known Newton-Raphson iterative method of function minimisation. Then, from a determined iteration $k$, we can approximate the next values of $\boldsymbol{X}$ from the last ones, using Eq. (3). Note that the solution we want to find is vector $\boldsymbol{X}$ of the unknowns, which solve Eq. (4).

$\boldsymbol{G}\left(\boldsymbol{X}_{k}\right) \approx \boldsymbol{G}\left(\boldsymbol{X}_{k-1}\right)+\boldsymbol{J}\left(\boldsymbol{X}_{k-1}\right) \Delta \boldsymbol{X}_{k}$,

$\boldsymbol{G}\left(\boldsymbol{X}_{k}\right)=0$.

Then Eq. (5) is derived, which may be solved from the least-squares method expressed in Eq. (6).

$\Delta \boldsymbol{X}_{k} \approx-\boldsymbol{J}^{-1}\left(\boldsymbol{X}_{k-1}\right) \boldsymbol{G}\left(\boldsymbol{X}_{k-1}\right)$

$\Delta \boldsymbol{X}_{k} \approx-\left(\boldsymbol{J}^{\mathrm{T}}\left(\boldsymbol{X}_{k-1}\right) \boldsymbol{J}\left(\boldsymbol{X}_{k-1}\right)\right)^{-1} \boldsymbol{J}^{\mathrm{T}}\left(\boldsymbol{X}_{k-1}\right) \boldsymbol{G}\left(\boldsymbol{X}_{k-1}\right)$.

If we consider that $n$ different correspondence matchings are obtained, then $\boldsymbol{G}\left(\boldsymbol{X}_{k-1}\right)$ is an $n$-dimensional vector. $\boldsymbol{J}\left(\boldsymbol{X}_{k-1}\right)$ is an $n \times 11$ matrix in which each column is a partial derivative of $\boldsymbol{G}$ from each unknown and each row and evaluation of these derivatives from each correspondence couple points. $\Delta \boldsymbol{X}_{k}$ is an 11-vector which contains the estimated error of the 11 unknown parameters to be determined. Eq. (6) is computed by the iterative algorithm. After each iteration, the error found in each parameter is added to its value obtaining the new value for the next iteration. We must iterate until Eq. (7) is reached.

$\Delta \boldsymbol{X}_{k}<\varepsilon$.

We have computed intrinsic and extrinsic parameters using the method proposed by Toscani. However, the maximum discrepancy between the modelled projections and the observable points is rather large as it was around 2.8 pixels. The main problem is due to the fact that Toscani does not take into account the lens deformation and that it is a non-iterative method. Then an iterative algorithm is quite interesting to decrease this discrepancy, readjusting the intrinsic and extrinsic parameters of the system. We have used the iterative method without modelling the lens deformation and we obtain a maximum error around 1.2 pixels, which improves considerably Toscani's method. Then, we have modelled the lens deformation. We start the iterative algorithm from the parameters obtained by Toscani and a null radial distortion. The focal distance $f$ has been fixed, otherwise the system is unstable because several 
Table 1

Intrinsic and extrinsic parameters obtained by the process of calibration

\begin{tabular}{|c|c|c|c|c|}
\hline & \multicolumn{2}{|c|}{ Camera parameters } & \multicolumn{2}{|c|}{ Projector parameters } \\
\hline & Initial solution & Iterative solution & Initial solution & Iterative solution \\
\hline$\alpha(\mathrm{rad})$ & 1.6605 & 1.7294 & 1.8091 & 1.7511 \\
\hline$\beta$ (rad) & -1.2051 & -1.2537 & -0.7090 & -0.7042 \\
\hline$\gamma(\mathrm{rad})$ & -3.0980 & -3.0325 & -2.9961 & -3.0324 \\
\hline$t_{x}(\mathrm{~mm})$ & 19.7 & -84.9 & -79.8 & -61.8 \\
\hline$t_{y}(\mathrm{~mm})$ & 144.9 & 106.3 & -82.7 & -14.3 \\
\hline$t_{z}(\mathrm{~mm})$ & 1829.4 & 2432.1 & 1772.1 & 1910.8 \\
\hline$f(\mathrm{~mm})$ & 10 & 10 & 10 & 10 \\
\hline$k_{u}(\mathrm{px} / \mathrm{mm})$ & 316.05 & 421.07 & 251.93 & 272.80 \\
\hline$k_{v}(\mathrm{px} / \mathrm{mm})$ & 332.57 & 442.43 & 261.31 & 282.66 \\
\hline$u_{\mathrm{o}}(\mathrm{px})$ & 223.43 & 405.19 & 376.22 & 350.82 \\
\hline$v_{\mathrm{o}}(\mathrm{px})$ & 227.25 & 297.80 & 560.48 & 460.09 \\
\hline$k_{1}(\mathrm{ad})$ & 0 & -0.03505 & 0 & -0.00309 \\
\hline
\end{tabular}

rad: radians; mm: millimetres; px: pixels; ad: adimensional

combinations of $\left(f, k_{u}, k_{v}\right)$ can be obtained without changing the $\left(\alpha_{u}, \alpha_{v}\right)$ values. That is why we have fixed $f=10 \mathrm{~mm}$. We have compared the initial solution with the final parameters. The maximum discrepancy between the modelled projections and the observable points with the obtained parameters was around 0.0053 and 0.0069 pixels for the camera and the projector system, respectively. Increasing the number of iterations, which is about the order of 20, does not improve the accuracy of the system. Table 1 shows the initial solution and the resulting intrinsic and extrinsic parameters obtained by the iterative algorithm.

A calibrating pattern made by two orthogonal planes have been set to calibrate the system (see Fig. 2). In order to calibrate the camera each plane is composed by a set of equidistant squares, obtaining a pattern like the one shown in Tsai (1987). The square vertexes are merely used to calibrate the camera. On the other hand, a millimetric sheet of paper is fixed on each calibrating plane in order to calibrate the projector. The coloured grid pattern is projected on both millimetric sheets of paper obtaining the 3D position of the slits intersections which are used to calibrate the projector.

\section{Pattern segmentation}

An RGB camera snaps an image of the pattern projected on the scene. The first step is the trans- formation of the image from the RGB model to the HSI perceptual model, close to human perception. In this way, the slits can be well segmented as we know the projected colour, and we have assumed that the scene is composed by pale and neutral colour objects. With this goal, we have used a realtime pre-processor composed by three (256 Kbytes) LUTs which are addressed by an 18 bits bus, 6 bits per each RGB channel, at video rate, obtaining the transformation from the three basic colours to the HSI model. Actually, LUTs are loaded with the hue, saturation and intensity values of the selected colours. The rest of the memory cells are all loaded with zeros. In fact, the pre-processor allows to deal with any mathematical colour conversion (the reader is referred to (Garcia-Campos et al., 1996) for a wide explanation of this card). Then, the HSI image containing the segmented scene is captured by a Matrox Meteor acquisition card into the memory of a PC Pentium.

At this point, we have three images corresponding to the previous chosen hue, saturation and intensity values into the memory of the personal computer. The six colours have been coded by six different grey levels in a single image. Then, this image has to be processed to detect straight segments for each grey level. We have used an easy methodology which involves image filtering, thinning and edge reconstruction. Fig. 3 shows the segmentation of the blue colour straight segments obtained after edge reconstruction of the first ex- 

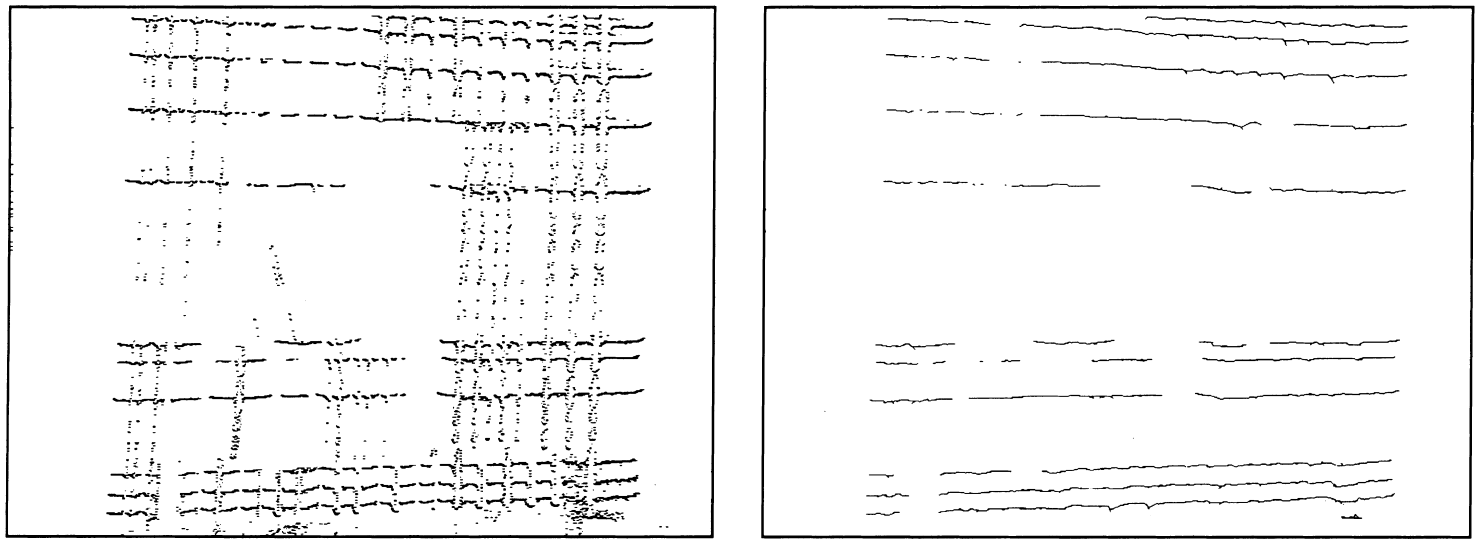

Fig. 3. Segmentation and edge reconstruction of blue colour.

perimental example. Straight segments will be useful to solve the correspondence problem. However, segments can not be used to detect the pattern cross-points due to their high discontinuity. Note that it is essential to obtain well segmented cross-points as they are directly used to infer 3D information.

The main idea is that cross-points have been detected by searching for local maximum in the intensity image. Actually, cross-points are made by the intersection of two slits with two different intensity levels. Then, the cross-point must have an intensity of approximately the sum of both which value defines a dynamic threshold. This threshold has been used to segment a region containing the cross-point which is further detected by computing the gravity centre. Fig. 4 shows the cross-point detection of an image portion of the first example.

Using the coloured codification of the segments which forms the cross-point in the image plane, the matching, i.e. the determination of the position of the same point in the projector image plane, is carried out. Then, 3D object point coordinates are obtained from both 2D positions of their projective points in the image plane and in the projected pattern.
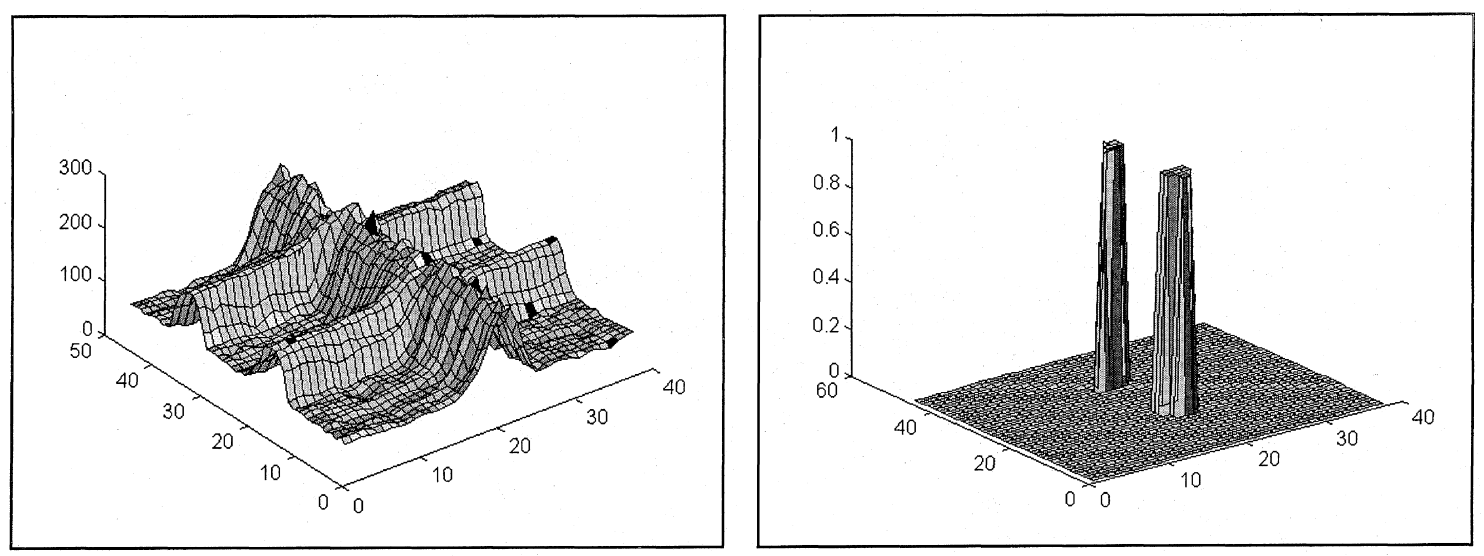

Fig. 4. A sample showing two cross-points detection. (a) A portion of the Intensity image showing three slits. (b) The segmented regions of both cross-points obtained by local thresholding. 


\section{Experimental results}

Hereafter, two examples are shown. The first example is composed by a scene with three geometric and achromatic objects illuminated by the coloured pattern, as shown in Fig. 5. Fig. 6 shows the measured correspondence points in the projector and camera frame, and Fig. 7 shows the 3D reconstruction. The second example is based on the 3D reconstruction of the mask shown in Fig. 8. Fig. 9 shows the $3 \mathrm{D}$ reconstruction.

In order to compare 3D geometric information inferred by the proposed coded structured light system and the real 3D information of the scene, we have projected the coloured pattern on a single plane. Several images have been taken while moving the plane. We have measured the $3 \mathrm{D}$ real point, and we have inferred the $3 \mathrm{D}$ object point from both projective $2 \mathrm{D}$ points. The $(X, Y, Z)$ error measured is the discrepancy between real coordinates and the inferred ones. We have measured the error in approximately 100 points. The average values of the error deviate $0.728 \mathrm{~mm}$ $(0.31 \%)$ from the $X$ axes, $0.624 \mathrm{~mm}(0.27 \%)$ from the $Y$ axes, and $0.465 \mathrm{~mm}(0.20 \%)$ from the $Z$ axes. The results are quite interesting if we take into account that the deviation degree is highly influenced by the segmentation process, i.e. by the
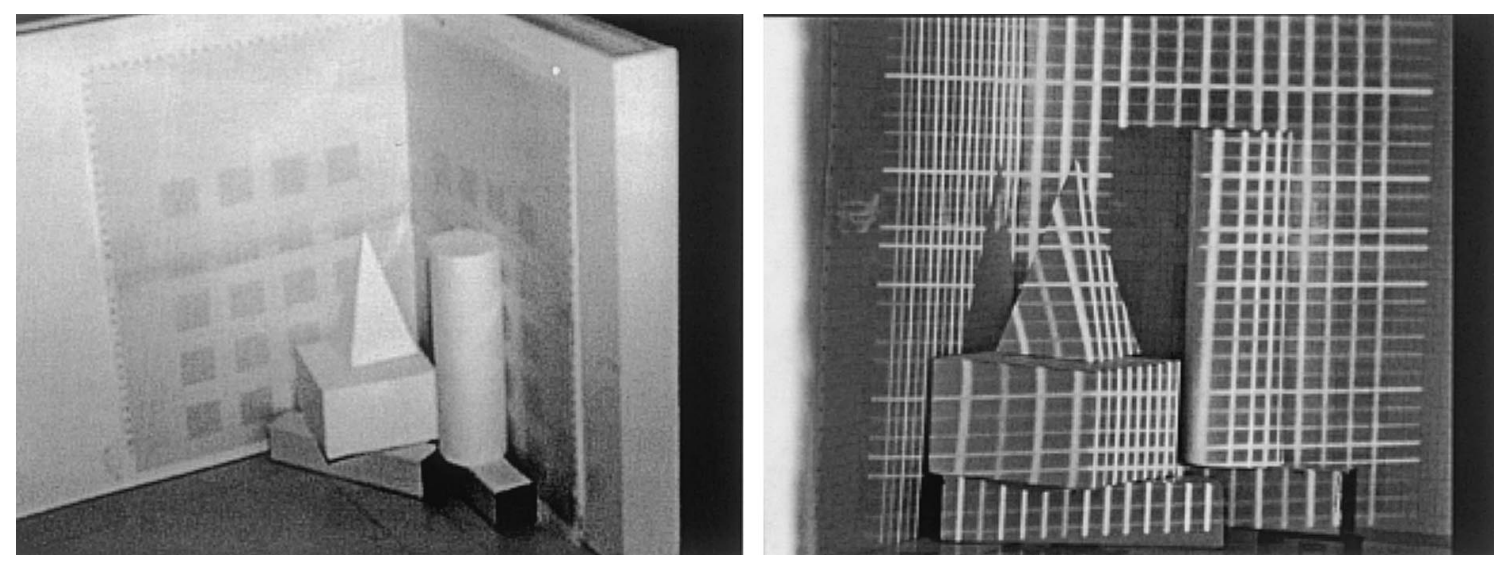

Fig. 5. (a) A real scene composed by three geometric objects (not used to infer 3D information). (b) The scene illuminated by the coloured pattern.
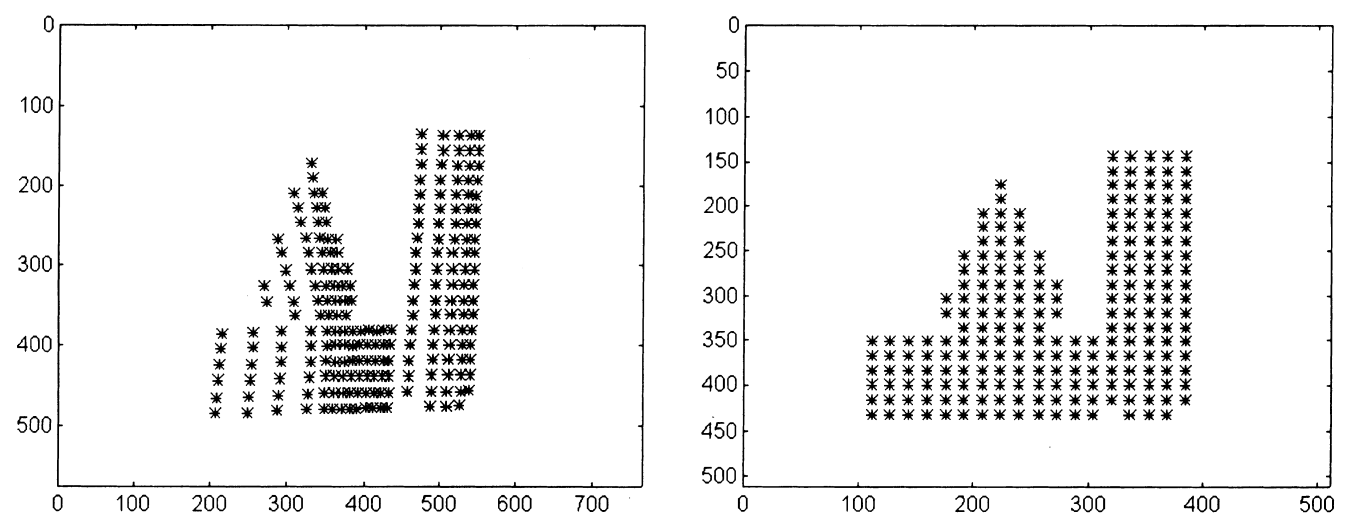

Fig. 6. The matching obtained from Fig. 5. (a) The Matching points from the $576 \times 768$ camera image plane. (b) The Matching points from the $512 \times 512$ projected pattern frame. 

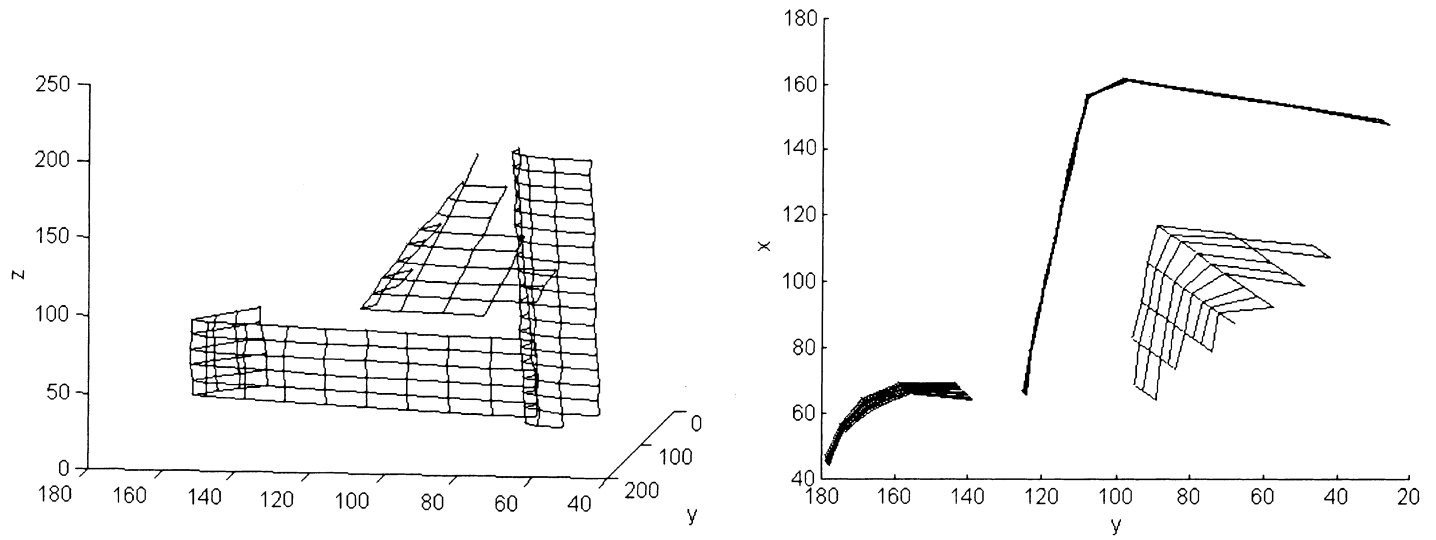

Fig. 7. The reconstruction of the $3 \mathrm{D}$ objects shown in Fig. 5. (a) A perspective view from the right side. (b) A top view.

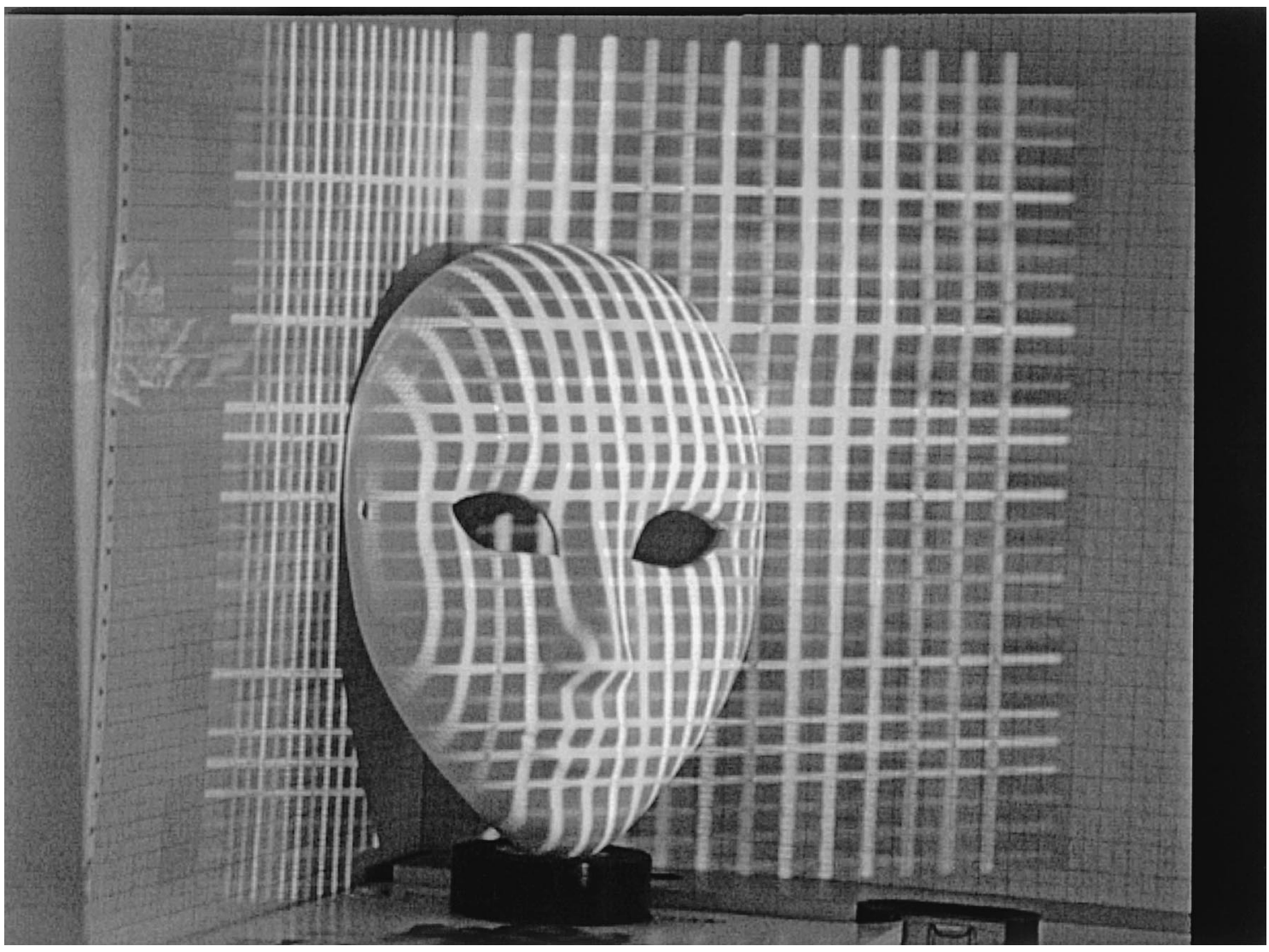

Fig. 8. A real scene composed by an illuminated mask. 

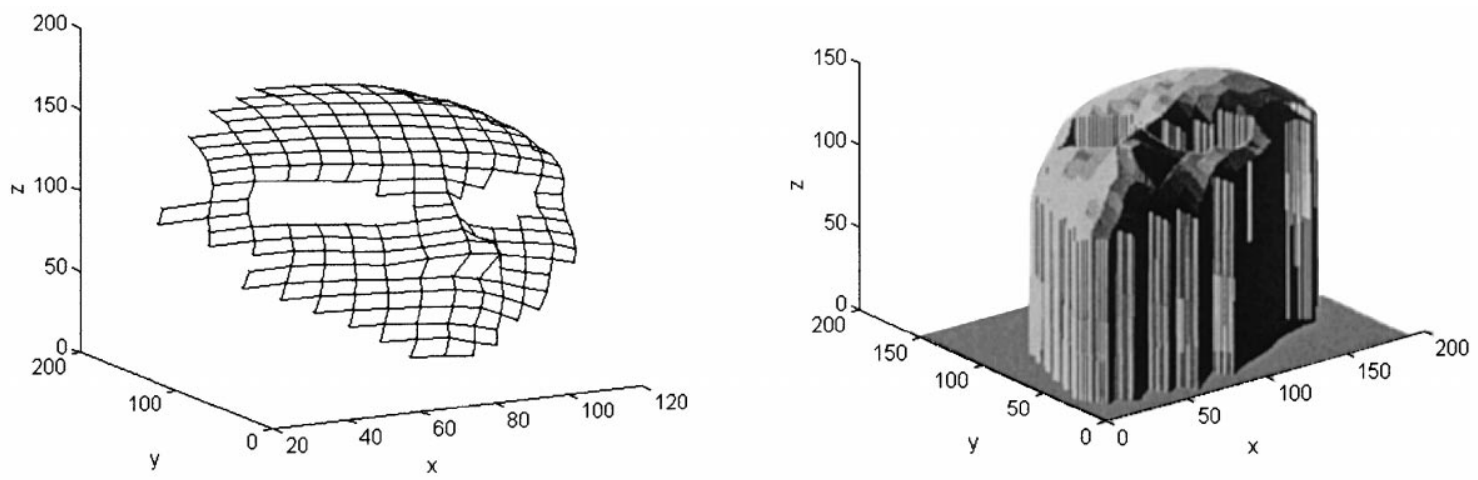

Fig. 9. The reconstruction of the mask shown in Fig. 8.

image noise and the problem of grabbing the projected lines of the pattern with different thickness.

On the other hand, the percentage of correctly identified intersections reaches the $100 \%$ of crosspoint identification, measuring a planar surface under scene light control. However, the identification is completely constrained by the kind of scene to be measured and the light conditions. Then, when the light conditions decrease, the projection of colour weaks considerably the use of such a kind of pattern. Of course, the utilisation of a digital light projector with a higher resolution, or even a more powerful lamp, will increase the average of correctly identified intersections.

\section{Conclusions}

In order to obtain a 3D measurement of either static or dynamic scenes, we propose to project a new coloured grid pattern on the measuring objects. The columns and rows of the grid are codified using six well-defined colours of the HSI cone. In the presented example, the pattern uses the three primary colours (red, green and blue) to code the rows. The magenta, cyan and yellow colours are used to code the columns. The slits have been coloured with the aim that each slit, with its two neighbours, forms a unique triplet in the whole pattern. Such a codification permits that the pattern could be used to obtain 3D information of the illuminated scene from a single shot projection.
Then, an easy and quick decoding step allows the pattern to be used in dynamic 3D measurements. Experimental results of object measurement and 3D reconstruction have been presented in the paper. Some applications of the sensor could, in particular, be used within the scope of scene interpretation in mobile robot navigation and tracking of moving objects, and, in general, in any $3 \mathrm{D}$ scene measurement where a single shot is allowed and a quick response of the system is required.

As a further work, we are interested in the frequency shifting of the imaging colour with respect to the projecting one as a result of the intrinsic colour of the measuring objects. If the pattern colours are well chosen, this discrepancy may allow us to obtain the intrinsic colour of the scene objects. Then the proposed system might be used to obtain 3D information and coloured information of any scene by a single pattern shot projection.

\section{References}

Batlle, J., Mouaddib, E., Salvi, J., 1997. A survey: Recent progress in coded structured light as a technique to solve the correspondence problem. To be published in Pattern Recognition Journal.

Boyer, K.L., Kak, A.C., 1987. Color-encoded structured light for rapid active ranging. Pattern Analysis and Machine Intelligence 9 (1), 14-28.

Faugeras, O., 1993. Three-Dimensional Computer Vision: A Geometric Viewpoint. MIT Press, London, England.

Garcia-Campos, R., Batlle, J., Bischoff, R., 1996. Architecture of an object-based tracking system using colour segmenta- 
tion. In: Proceedings of 3rd Internat. Workshop on Image and Signal Process., pp. 299-302.

Griffin, P.M., Narasimhan, L.S., Yee, S.R., 1992. Generation of uniquely encoded light patterns for range data acquisition. Pattern Recognition 25 (6), 609-616.

Hall, E.L., Tio, J.B.K., McPherson, C.A., Draper, C.S., Sadjadi, F.A., 1982. Measuring curved surfaces for robot vision. Computer (December), 42-54.

Hu, G., Stockman, G., 1989. 3-D surface solution using structured light and constraint propagation. Pattern Analysis and Machine Intelligence 11 (4), 390-402.

Ito, M., Ishii, A., 1995. A three-level checkerboard pattern (TCP) projection method for curved surface measurement. Pattern Recognition 28 (1), 27-40.

Maruyama, M., Abe, S., 1993. Range sensing by projecting multiple slits with random cuts. Pattern Analysis and Machine Intelligence 15 (6), 647-651.

Tajima, J., Iwakawa, M., 1990. 3-D data acquisition by rainbow range finder. In: Proceedings of IEEE Internat. Conf. on Pattern Recognition, pp. 309-313.
Toscani, G., 1987. Systèmes de calibration et perception du mouvement en vision artificielle. Thèse Université Paris Sud.

Tsai, R.Y., 1987. A versatile camera calibration technique for high-accuracy $3 \mathrm{D}$ machine vision metrology using off-theshelf TV cameras and lenses. Journal of Robotics and Automation RA-3 (4), 323-344.

Vuylsteke, P., Oosterlinck, A., 1990. Range image acquisition with a single binary-encoded light pattern. Pattern Analysis and Machine Intelligence 12 (2), 148-164.

Wang, Y.F., Pandey, A., 1991. A study on using structured lighting to analyze time varying image seguences. Pattern Recognition 24 (8), 723-738.

Weng, J., Cohen, P., 1992. Camera calibration with distortion models and accuracy evaluation. Pattern Analysis and Machine Intelligence 14 (10), 965-980.

Will, P.M., Pennington, K.S., 1971. Grid coding: A preprocessing technique for robot and machine vision. Computers and Artificial Intelligence, pp. 66-70.

Yee, S.R., Griffin, P.M., 1994. Three-dimensional imaging system. Optical Engineering 33 (6), 2070-2075. 